\title{
使用卫星雷达差分干涉技术测量 香港赤腊角机场沉降场
}

\author{
刘国祥 ${ }^{(1)}$ 丁晓利 ${ }^{(2)}$ 陈永奇 ${ }^{(2)}$ 李志林 $^{(2)}$ 李志伟 $^{(2)}$
}

(1)西南交通大学土木工程学院测量工程系, 成都 610031; 2)香港理工大学土地测量及地理资讯学系, 香港.

E-mail: gx.liu@polyu.edu.hk)

\begin{abstract}
摘要 使用卫星雷达差分千涉技术考察建立在近海回填地基上的香港赤腊角机场的稳定性. 利用 ERS-2 卫星获取的合成孔径雷达影像作差分千涉处理, 获取了该机场在近乎一年内的非均匀沉降场, 地 面分辨率为 $20 \mathrm{~m} \times 20 \mathrm{~m}$, 在填海区域内下沉量呈 0 50 mm 的空间分布, 与离散水准监测结果相比吻合 较好(相关系数 0.89). 特别地, 一个被开挖岛屿的原轮廓可从千涉结果中分辨出来. 同时, 探讨了区分 和估计千涉失相关和大气延迟影响的有效方法. 研究证实了 ERS-2 千涉系统对微小的地表沉降具有很 高的敏感度, 结果精度优于 $1 \mathrm{~cm}$.
\end{abstract}

\section{关键词 合成孔径雷达差分干涉 干涉失相关 大气延迟 填海 沉降场}

近 10 年来, 一个新的遥感技术一一合成孔径雷达 干涉(synthetic aperture radar interferometry, InSAR) 在理论和应用上正迅速发展，它具有高精度、高分辨 率和连续空间覆盖等良好的技术优势. 特别地，使用 多幅雷达图像且围绕其相位信号处理的差分干涉 (differential InSAR, D-InSAR)方法, 可用于大规模监 测厘米级甚至更小的地球表面形变, 以揭示许多地 球物理现象, 如板块运动 ${ }^{[1]}$ 、地震形变 ${ }^{[2 \sim 4]}$ 、火山运 动 ${ }^{[5]}$ 、冰川漂移 ${ }^{[6]}$ 、地面下沉 ${ }^{[7]}$ 以及山体滑坡 ${ }^{[8]}$ 等. 国 内外地学科学家普遍期望, D-InSAR 能为地球物理动 态过程的研究提供一种全新的途径 ${ }^{[2,5,6]}$. 目前, 干涉 相位失相关 ${ }^{[9]}$ 仍然是监测缓慢累积形变(如沉降)的主 要障碍, 潜在的大气(特别是水汽含量的时空变化)延 迟的影响 ${ }^{[10]}$ 有时会制约目的信息的提取，因而，国 际上大范围监测地面垂直位移的成功范例并不多

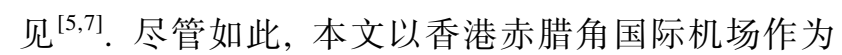
研究对象，使用该技术探测到了其缓慢的沉降信息. 赤腊角机场于 1998 年 7 月启用, 它建立在一个人工 岛上, 总面积为 $12.48 \mathrm{~km}^{2}, 75 \%$ 为填海地基, $25 \%$ 为 开挖两个小岛所得 (见图 1), 常规地面水准监测已显 示填海地基仍处于下沉期, 但受空间分辨率的限制, 很难详细地反映该沉降场 ${ }^{[11]}$. 我们使用欧洲空间局 的 ERS-2 卫星分别于 1998 年 12 月 29 日和 1999 年 11 月 9 日在香港上空重复通过获取的两景 SAR 影像 进行干涉处理, 分析干涉相位的噪声特性和大气影 响，并提取该机场在近乎一年内的累积沉降分布。最
后对其沉降机制作出解释.

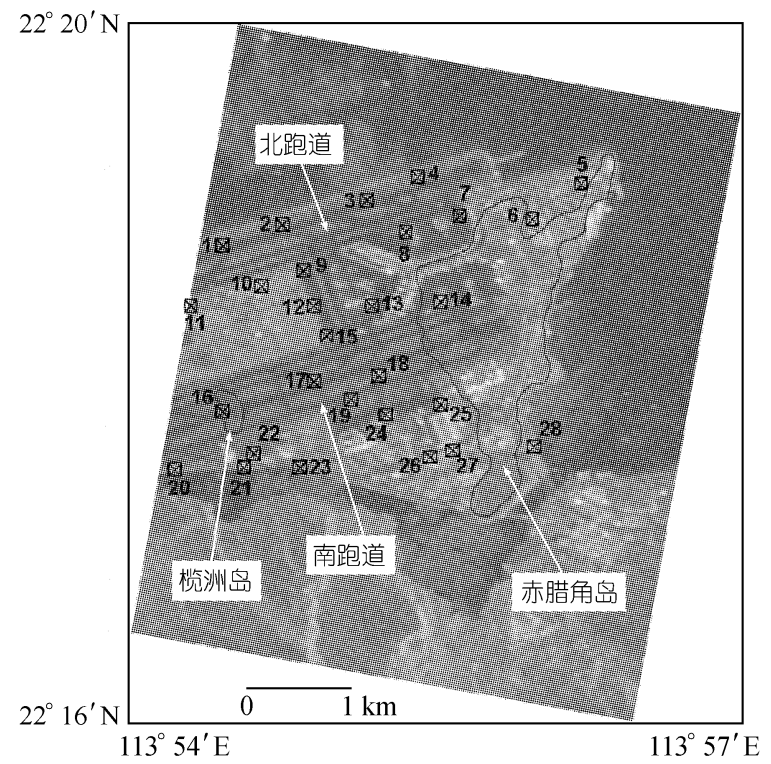

图 1 赤腊角机场 ERS-2 SAR 图像

28 个地面水准监测点和被开挖的两个小岛原区域的边界被叠加显示

\section{1 差分干涉沉降监测方法}

关于 D-InSAR 的观测几何和基本原理，其他地 方已有详细的陈述 ${ }^{[2-5,12]}$, 这里重点讨论利用它提取 地表沉降信息的方法和分析其敏感度. 图 2 显示了 ERS-2 卫星重复轨道干涉系统的几何配置. 设卫星在 轨道点 $S_{1}$ 和 $S_{2}$ 位置先后成像的时间间隔内, 某一地 
面分辨元 $P$ 沉降了 $\Delta h$ 且移动到 $P^{\prime}$, 两次成像的雷达 斜距分别为 $R_{1}$ 和 $R_{2}$, 由于 $B<<R_{i}(i=1,2)$, 故可认为 雷达斜距相互平行. 实际上, 因沉降致使 $S_{2}$ 的雷达斜 距发生了改变, 即将沉降信息附加到观测相位之中. 两次观测所得的一次差分相位 $\delta \phi$ 归结为 3 种贡献: ( i ) 地球参考椭球面, (ii) 地形起伏即相对于椭球面 的高程和 (iii) 地表沉降. 与此相对应的雷达斜距差可 分解为 3 部分, 即图中所示的 $\delta s, \delta t$ 和 $\delta d$. 值得指出的 是因该机场平台总体高程在 $6.0 \mathrm{~m}$ 左右, 因此 $\delta s$ 与 $\delta t$ 之和近似为基线 $B$ 在 $R_{2}$ 上的投影.

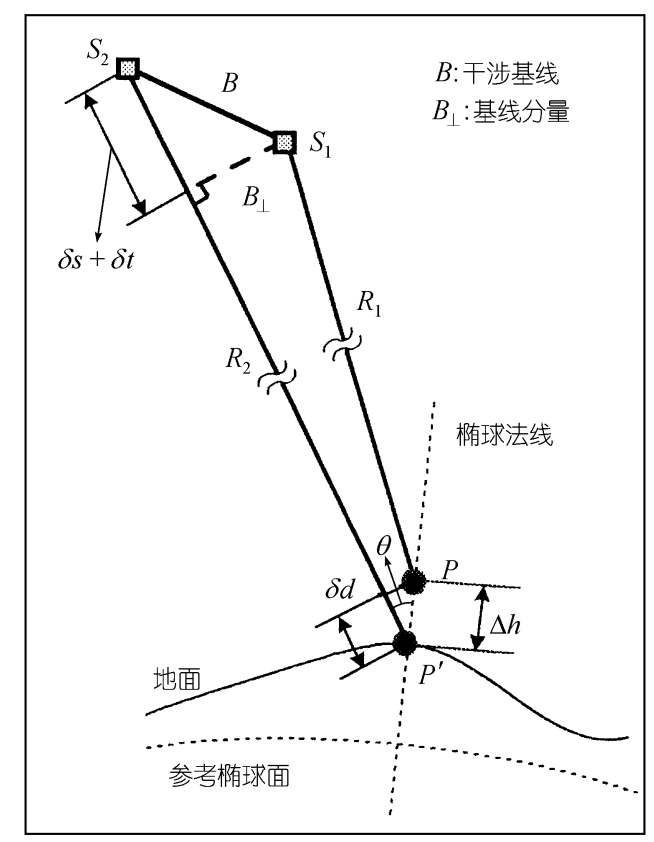

图 2 ERS-2 重复轨道干涉几何

为了提取沉降信息, 首先借助于卫星轨道数据、椭 球模型及成像几何去除椭球系统相位 ${ }^{[3]}$, 进而利用数 字高程模型、轨道数据和雷达系统参数模拟地形相位 并去除 ${ }^{[5]}$ (即二次差分), 则残余差分相位 $\Delta \phi$ 对应着 雷达斜距改变量 $\delta d$, 其关系为

$$
\delta d=\frac{\lambda \cdot \Delta \phi}{4 \pi},
$$

式中, $\lambda$ 为雷达波长 $(\mathrm{ERS}-2 \mathrm{SAR}$ 使用 $\mathrm{C}$ 波段, 波长为 $5.6 \mathrm{~cm})$. 因此沉降量 $\Delta h$ 可如下得到:

$$
\Delta h=\frac{\delta d}{\cos \theta}=\frac{\lambda \cdot \Delta \phi}{4 \pi \cdot \cos \theta},
$$

式中, $\theta$ 为相对于椭球法线的雷达人射角(ERS-2 SAR 的名义值为 $23^{\circ}$ ). 由(2)式可知, $\mathrm{ERS}-2 \mathrm{SAR}$ 差分干涉
对沉降的敏感度为 $3.0 \mathrm{~cm}$, 即一个整周相位 $2 \pi$ 可测 定 $3.0 \mathrm{~cm}$ 的沉降量. 设相位观测误差为 $20^{\circ}$, 沉降量 $\Delta h$ 的理论精度为 $1.7 \mathrm{~mm}$, 这说明了使用短波段的 ERS-2 SAR 干涉系统对微小的地表沉降相当敏感. 值 得说明的是, (1)式中的 $\Delta \phi$ 存在整周模糊度, 这是相位 测距普遍存在的问题, 需利用相位解缠算法 ${ }^{[13]}$ 来 确定.

\section{2 数据处理和干涉结果}

由于赤腊角机场平台相当平坦且有香港地政总 署测绘处提供的数字高程模型(高程精度为 $1.0 \mathrm{~m}$ ) 可 利用，因而两通差分干涉方法被采用. 首先将前已述 及的两幅 SAR 图像数据作空间配准 ${ }^{[14]}$ 且将一幅影像 取样到另一幅影像空间 ${ }^{[3]}$, 再将对应像素作复数共轭 相乘, 则得到初始相位干涉图, 然后作椭球系统相位 去除和低通滤波以平滑相位噪声(多视平滑窗口为 $1 \times$ 5), 同时测度干涉相位可靠性的相关图 ${ }^{[5]}$ 也一并算出. 为综合展示干涉质量, 将雷达灰度影像、计算所得的 一次差分干涉图和相关图运用彩色合成方法将它们叠 加在一起. 图 3 显示了这 3 种信息融合后关于赤腊角 机场和大屿山岛地区约 $425 \mathrm{~km}^{2}$ 的干涉影像, 地面分 辨率为 $20 \mathrm{~m} \times 20 \mathrm{~m}$. 很明显, 在海面上没有任何干 涉条纹, 山区干涉条纹也较为模糊, 而机场区域呈现 较好的干涉相关性(平均相关系数为 0.31). 因镜面反 射或海潮的影响, 海面干涉信号基本上完全失相关. 因雷达先后成像时间间隔较长(315 d), 山区的植被会 变化、成像时风对植被的影响或地表湿度变化等因素 都会致使雷达分辨元的后向散射特性产生随机性改 变, 故雷达信号相关性显著降低. 但机场几乎没有植 被覆盖, 地表物理特性 (如介电常数)相对稳定, 即使 $315 \mathrm{~d}$ 的成像间隔, 雷达信号的相关性仍维持得较好, 为形变探测提供了保证. 图 3 中一个干涉条纹表示了 一个整周干涉相位 $0 \rightarrow 2 \pi$ 的变化. 若无地表形变存在, 在垂直基线分量 $B_{\perp}$ 为 $481.5 \mathrm{~m}$ 的条件下(如图 2 所示), 一个整周相位变化对应 $22.2 \mathrm{~m}$ 的地表高程变化.

二次差分处理的焦点集中在机场区域. 首先利用 机场已有的数字高程模型、卫星轨道数据和雷达系统 参数模拟地形相位, 再从上述一次差分相位图中扣除 地形的影响. 图 4 放大地显示了机场区域的二次差分 干涉相位图. 因参考椭球和地形贡献的相位已被去除 掉, 残余相位与机场平台的不均匀沉降分布有关, 故 此时相位图中的每 - 像素的相位值仍存在整周模糊 


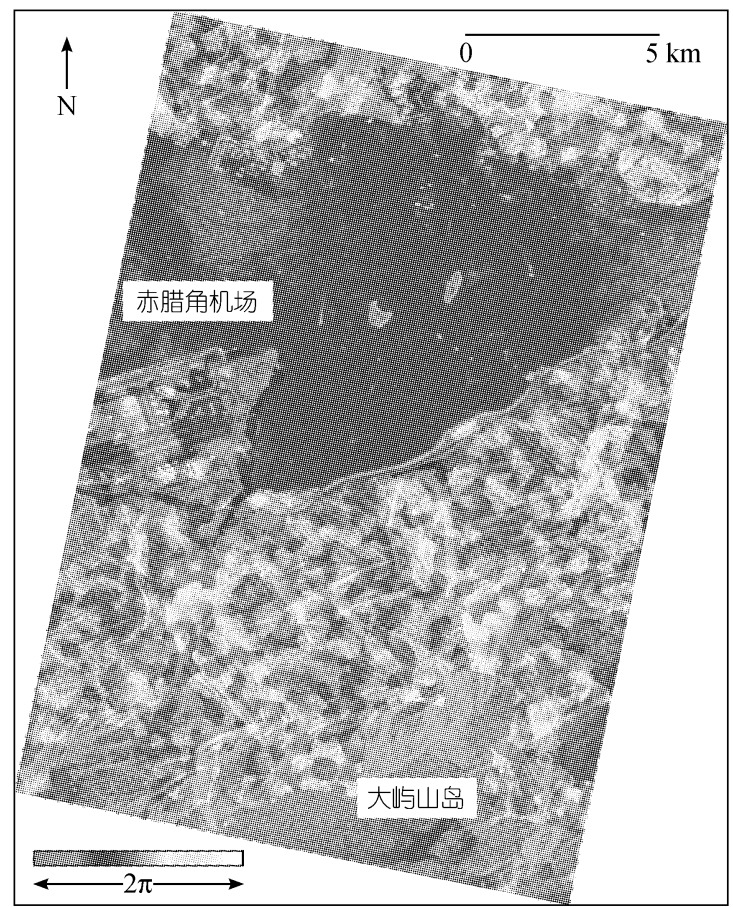

图 3 雷达灰度影像、一次差分干涉相位图和 相关影像叠加结果

$B_{\perp}=481.5 \mathrm{~m}, h_{2 \pi}=22.2 \mathrm{~m}$

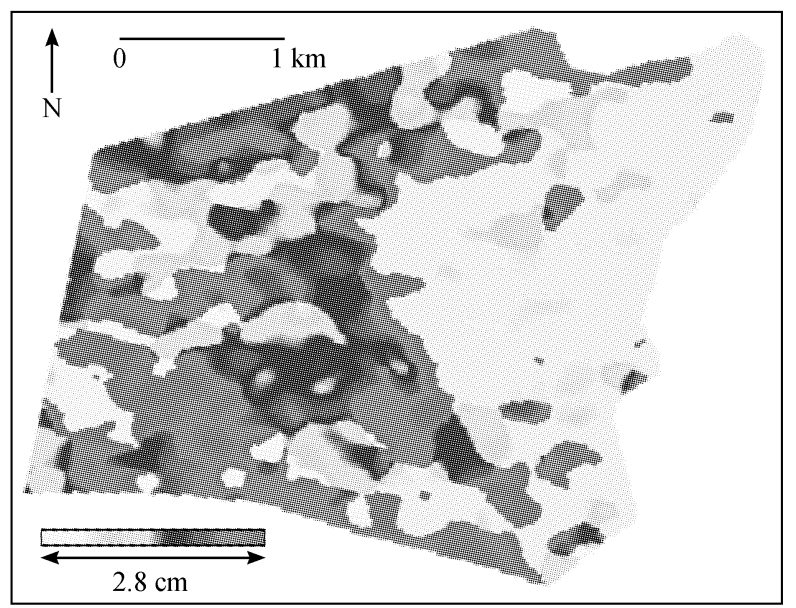

图 4 赤腊角机场的二次差分干涉相位图 一个干涉条纹相当于 $2.8 \mathrm{~cm}$ 的雷达斜距改变量的变化

度. 我们使用基于路径控制的积分算法 ${ }^{[13]}$ 对相位图进 行解缠(令图 1 中 14 号水准点的相位为 0 , 作为解缠的 起点), 以确定任意像素点间相位的相对关系. 借助 (1)和(2)式逐像素计算沉降量, 并投影到 HK80 格网坐 标系统下, 最后结果如图 5 所示/ 沉降量以分级的色
调来表示，在填海区域内，地表下沉量具有明显空间 变化(0 50 mm). 在开挖区域即原两个小岛赤腊角 $\left(3.02 \mathrm{~km}^{2}\right)$ 和榄洲 $\left(0.08 \mathrm{~km}^{2}\right)$ 的范围内(见图 1), 无显著 的沉降发生, 其稳定性可从图 4 和 5 中可见. 特别地, 赤腊角岛的原轮廓大致地但十分清晰地反映在差分干 涉图中. 值得指出的是, DEM 不确定性 $( \pm 1.0 \mathrm{~m})$ 引人 的估计误差 ${ }^{[10]}$ 为 $1.3 \mathrm{~mm}$, 而轨道数据不确定性引人 的误差呈系统性(微小倾斜). 对于这个小的研究区域 来说, 相对误差是可忽略的 ${ }^{[3]}$.

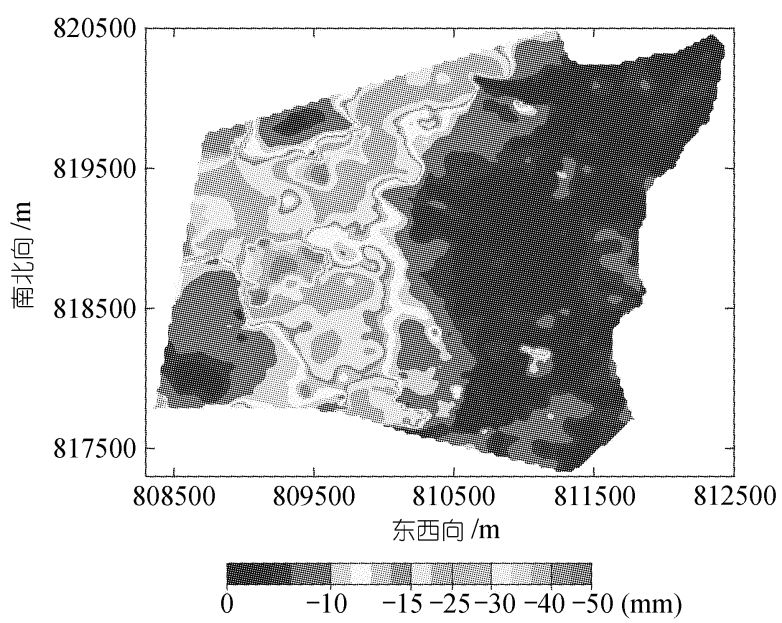

图 5 赤腊角机场 $315 \mathrm{~d}$ 内累积的沉降场

另一方面，机场管理局一直在使用地面一等精密 水准方法监测机场沉降. 我们得到了 28 个水准点在这 $315 \mathrm{~d}$ 内(干涉时间间隔)的沉降结果，以 SAR 灰度影像 作背景. 这些水准点的分布显示在图 1 中. 为证实干 涉结果的可靠性, 这 28 个点的干涉结果被提取出来与 水准结果作比较. 表 1 显示了这两类观测结果的差异 情况, 两类结果的总体相关系数为 0.89 , 差异均值为 -3.5 mm, 差异的总体标准偏差为 $5.6 \mathrm{~mm}$. 这表明了 干涉结果的精度较高, 总体精度优于 $1 \mathrm{~cm}$, 而误差的 主要来源是干涉失相关引人的噪声或潜在的大气相位 延迟的影响. 对处于热带和近海的香港地区来说, 雷 达差分干涉技术用于监测缓慢累积的微小地表沉降受 干涉失相关和可能的大气变化影响两大因素的制约更 为显著. 进一步, 我们通过理论估计方差和经验统计 方差的对比来分析差分干涉相位的随机性和系统性, 以此考察干涉失相关和可能的大气影响. 被开挖的两 个岛屿具有坚硬的花岗岩地质条件 ${ }^{[11]}$ ，设这两个区域 没有发生任何沉降, 则其非缠绕的残余相位仅与失相 
关引人的噪声和可能的大气相位延迟相关. 这两个独 立的样本区域的平均干涉相关系数分别为 0.29 和 0.32 , 根据理论方差估计公式 ${ }^{[9]}$ 可得它们的相位中误 差分别为 1.04 和 0.94 弧度, 而经验统计方差分别为 1.13 和 0.82 弧度. 这两组数据非常接近, 这会导致沉 降测量误差在 $5.4 \mathrm{~mm}$ 左右, 与干涉-水准较差的标准 偏差 $5.6 \mathrm{~mm}$ 也非常一致. 因而可以推测, 空间尺度上 的大气变化引人的相位延迟的系统性影响很弱, 而失 相关的随机噪声影响相对占主导地位. 这种比较逻辑 应用在其他干涉图的分析中也是有效的. 通过取样分 析, 可以估计大气的影响在图 5 所示干涉结果中可以 忽略, 而干涉失相关适当降低了干涉结果的可靠性.

\section{表 $1 \quad$ InSAR 与水准监测结果差异比较}

\begin{tabular}{|c|c|c|c|}
\hline 点号 & 水准沉降 / mm & 干涉沉降/ mm & 差异/ mm \\
\hline 1 & -20.0 & -15.1 & -4.9 \\
\hline 2 & -10.0 & -10.3 & 0.3 \\
\hline 3 & -32.0 & -22.4 & -9.6 \\
\hline 4 & -36.0 & -24.2 & -11.8 \\
\hline 5 & -5.0 & -3.2 & -1.8 \\
\hline 6 & -5.0 & -1.8 & -3.2 \\
\hline 7 & -3.0 & -0.4 & -2.6 \\
\hline 8 & -15.0 & -11.0 & -4.0 \\
\hline 9 & -55.0 & -37.4 & -17.6 \\
\hline 10 & -32.0 & -36.1 & 4.1 \\
\hline 11 & -20.0 & -26.0 & 6.0 \\
\hline 12 & -21.0 & -22.1 & 1.1 \\
\hline 13 & -13.0 & -10.1 & -2.9 \\
\hline 14 & -1.0 & -0.8 & -0.2 \\
\hline 15 & -27.0 & -15.3 & -11.7 \\
\hline 16 & -1.0 & -4.6 & 3.6 \\
\hline 17 & -32.0 & -25.0 & -7.0 \\
\hline 18 & -6.0 & -8.5 & 2.5 \\
\hline 19 & -23.0 & -13.0 & -10.0 \\
\hline 20 & -26.0 & -19.4 & -6.6 \\
\hline 21 & -30.0 & -19.2 & -10.8 \\
\hline 22 & -17.0 & -18.5 & 1.5 \\
\hline 23 & -20.0 & -13.1 & -6.9 \\
\hline 24 & -11.0 & -8.7 & -2.3 \\
\hline 25 & -3.0 & -3.0 & 0.0 \\
\hline 26 & -10.0 & -10.8 & 0.8 \\
\hline 27 & -9.0 & -10.1 & 1.1 \\
\hline 28 & -6.0 & -2.5 & -3.5 \\
\hline
\end{tabular}

\section{3 沉降解释与结论}

从地质上来说, 赤腊角机场填海区域属于第四纪 序列, 从上至下包括非均匀的海泥层、冲积层(依次可 细分为黏土层、泥沙层和砂砾层)和风化岩石层等. 在
填海工程开始之前, 填海区域 $\left(9.38 \mathrm{~km}^{2}\right)$ 的海泥层已 被最大限度地挖走. 当海泥层清理掉后, 相对于海平 面, 海床起伏在 $-3 \sim-29 \mathrm{~m}$ 间变化, 海床深度由南向 北逐渐变大. 填海所用材料为从两个小岛开挖的石 料、从其他地方运来的石料和海沙等. 因填方荷载的 引人, 填海地基受 3 种主要沉降机制的影响: ( i ) 短 时间内黏土层、泥沙层的显著性一级压缩，（ii）缓慢 的砂砾层二级压缩，(iii）填料层的自压缩. 因为在 1998 年 12 月 29 日获取第 1 个 SAR 图像时, 黏土层和 泥沙层的一级压缩已基本完成 ${ }^{[11]}$, 故图 5 所示的干涉 沉降场主要源于后两种沉降机制的贡献，而非均匀的 下沉量空间分布与回填区域内地质条件和填方厚度具 有显著的空间变化特征相关，如机场西北区域内存在 快速的空间变化(见图 4 和 5).

以近海回填区域赤腊角机场平台为研究对象，通 过对 ERS-2 SAR 数据的差分干涉处理提取到了该机 场在运营后将近一年内的累积沉降分布, 空间分辨率 为 $20 \mathrm{~m}$, 在填海区域内, 地表下沉量具有明显空间变 化(0 50 mm). 特别地, 被开挖的赤腊角岛原轮廓可从 干涉结果中分辨出来. 利用离散水准监测资料证实了 干涉结果的可靠性, 精度优于 $1 \mathrm{~cm}$. 通过我们的比较 逻辑, 证实了该干涉结果受大气的影响可忽略, 而干 涉失相关的影响相对占主导地位. 理论分析和实际应 用证明了使用短波段的 ERS-2 雷达干涉系统对微小的 地表沉降具有很高的敏感性. 特别地，其高空间分辨 率的技术优势是其他已有监测技术难以婫美的. 随着 干涉技术的进一步发展，它将为大范围的近海回填地 基的稳定性监测提供廉价的、可靠的技术支持，与常 规的地面监测方法相配合可大大地改善沉降场的预报 模型，为基础设施和建筑物的维护与进一步规划提供 依据. 此外, 差分干涉结果又可为地面沉降监测网的 优化设计与扩展提供重要的参考.

致谢第一作者感谢香港理工大学提供的博士生奖学金. 本工作为香港理工大学建设与地政学院的 “系统合作研 究” 项目、香港理工大学研究设备基金(批准号: G-9025) 和研究基金(批准号: G-V747)资助项目.

\section{参考文献}

1 Fujiwara S, Rosen P A, Tobita M, et al. Crustal deformation measurements using repeat-pass JERS-1 synthetic radar interferometry near the Izu Peninsula, Japan. J Geophys Res, 1998, 103: $2411 \sim 2426$ 
2 Massonnet D, Rossi M, Carmona C, et al. The displacement field of the landers earthquake mapped by radar interferometry. Nature, 1993, 364: 138 142

3 Zebker H A, Rosen P A, Goldstein R M, et al. On the derivation of coseismic displacement fields using differential radar interferometry: The landers earthquake. J Geophys Res, 1994, 99: 19617 $\sim 19634$

4 王超, 刘智, 张红, 等. 张北-尚义地震同震形变场雷达差分干 涉测量. 科学通报, 2000, 45(23): 2550 2554

5 Rosen P A, Hensley S, Zebker H A, et al. Surface deformation and coherence measurements of Kilauea Volcano, Hawaii, from SIR-C radar interferometry. J Geophys Res, 1996, 101: 33109 33135

6 Goldstein R M, Engelhardt H, Kamb B, et al. Satellite radar interferometry for monitoring ice sheet motion, application to an Antarctic ice stream. Science, 1993, 262: 1525 1530

7 Galloway D L, Hudnut K W, Ingebritsen S E, et al. Detection of aquifer system compaction and land subsidence using interferometric synthetic aperture radar, Antelope Valley, Mojave Desert, California. Water Resources Research, 1998, 34: 2573 2585
8 Kimura H, Yamaguchi Y. Detection of landslide areas using satellite radar interferometry. Photogrammetric Engineering \& Remote Sensing, 2000, 66: 337 344

9 Zebker H A, Villasenor J. Decorrelation in interferometric radar echoes. IEEE Trans Geosci Remote Sens, 1992, 30(5): 950 959

10 Zebker H A, Rosen P A, Hensley S. Atmospheric effects in interferometric synthetic aperture radar surface deformation and topographic maps. J Geophys Res, 1997, 102: 7547 7563

11 Plant G W, Covil C S, Hughes R A. Site Preparation for the New Hong Kong International Airport. London: Thomas Telford Publishing Ltd, 1998. 150 256

12 刘国祥, 丁晓利, 陈永奇, 等. 极具潜力的空间对地观测新技术: 合成孔径雷达干涉. 地球科学进展, 2000, 15(6): 734 740

13 Goldstein R M, Zebker H A, Werner, C L. Satellite radar interferometry: two-dimensional phase unwrapping. Radio Science, 1988, 23: 713 720

14 刘国祥, 丁晓利, 李志林, 等. 星载 SAR 复数图像的配准. 测绘 学报, 2001, 30(1): 60 66

(2001-03-09 收稿, 2001-06-14 收修改稿) 